\title{
Microphonics in Biopotential Measurements with Capacitive Electrodes
}

\author{
Pablo S. Luna-Lozano Student Member, Ramon Pallas-Areny, Fellow, IEEE
}

\begin{abstract}
Biopotential measurements with capacitive electrodes do not need any direct contact between electrode and skin, which saves the time devoted to expose and prepare the contact area when measuring with conductive electrodes. However, mechanical vibrations resulting from physiological functions such as respiration and cardiac contraction can change the capacitance of the electrode and affect the recordings. This transformation of mechanical vibrations into undesired electric signals is termed microphonics. We have evaluated microphonics in capacitive ECG recordings obtained from a dressed subject seated on a common chair with electrodes placed on the front side of the backrest of the chair. Depending on the softness of the backrest, the recordings may be clearly affected by the displacement of the thorax back wall due to the respiration and to the heart's mechanical activity.
\end{abstract}

\section{INTRODUCTION}

C OnVEnTIONAL surface biopotential measurements require the exposure and preparation of the skin areas where electrodes are to be placed, and/or the use of an electrolyte. Dry-electrodes do not need any electrolyte nor skin preparation but still need the exposure of the body contact areas. Off-body biopotential measurements have been tried [1], [2] that do not need any contact with the body, but the actual origin of the recorded signals is disputed because electrostatic charges and distance variations between the body and the electrodes can result in signals larger than surface biopotentials, hence not attributable to them [3], [4].

Strapping electrically insulated metal electrodes to the body implies a mechanical contact that establishes a fixed distance between the body and the metal part of the electrode, thus offering more reliable signals [5]. An alternative technique is to detect bioelectric signals through the subject's clothes by using electrodes placed on an object that comes into contact with his/her body [6], [7], [8]. A capacitor is formed between the skin and the electrode metal, and the electrically-insulating clothes become the capacitor's dielectric. This approach frees the user from servitudes such as battery replacement in portable devices and avoids any cumbersome electrodes or wires. However, distance variations between capacitor plates (the skin and the electrode) can result in artifacts [9]. Those distance

Manuscript received June 26, 2010. This work was supported by the Spanish Ministry of Education and Science under contract TEC2009-13022, and the European Regional Development Fund. P. S. Luna is a PhD student supported by a scholarship (Ref. 166345) from CONACyT, Mexico.

P. S. Luna and R. Pallas-Areny are with the Instrumentation, Sensors and Interfaces Group, Castelldefels School of Technology, Universitat Politècnica de Catalunya (UPC), Castelldefels (Barcelona), 08860, Spain (email: 1una@eel.upc.edu, ramon.pallas@upc.edu). variations can result from relatively large voluntary movements but also from physiological movements such as those related to the breathing and heart activity. In this work we analyze the origin, relevance and reduction of those physiologically-induced movement artifacts.

\section{MICROPHONICS IN MEASUREMENTS WITH CAPACITIVE ELECTRODES}

Microphonics is any electrical interference caused by mechanical vibration. When a metal electrode is placed on a seat's backrest and a dressed person comes into mechanical contact with it, thoracic movements due to respiration and heart contractions may change the actual distance between the body and the electrode (see Fig. 1). In each cardiac cycle, atrial and ventricular contractions make the chest wall to move as recorded in the apexcardiogram (ACG) [10]. Further, the heart contraction and blood ejection by the left ventricle, and the subsequent blood mass flow up in the ascending aorta and down in the descending and abdominal aortas, make the whole body to move, as revealed by the ballistocardiogram (BCG) [10].

When attempting to record the electrocardiogram (ECG) with capacitive electrodes, the absence of any conductive contact between the body and the electrodes makes it necessary to provide a return path for input bias currents of amplifiers connected to the electrodes. Further, to reduce power line interference coupling to the electrodes and to wires connecting them to amplifiers, each electrode is usually connected to a voltage buffer close to it and whose input is connected to signal ground through a large bias resistor $\left(R_{\mathrm{B}}=100 \mathrm{G} \Omega\right.$ in [7]) that will be shunted by the buffer's input capacitance $C_{\mathrm{i}}$. Thus, the equivalent capacitance $C_{\mathrm{e}}$ between the body and the (capacitive) electrode form a high-pass filter (HPF) with $R_{\mathrm{B}}$, and any variation in $C_{\mathrm{e}}$ will translate into changes in the filter's corner frequency $f_{\mathrm{c}}$. If $f_{\mathrm{c}}$ is not small enough compared to the spectral contents of the ECG, the voltage recorded will be attenuated by a factor whose value will depend on those distance variations. Normally, $C_{\mathrm{e}}$ is very small because of the limited electrode area and the thickness of clothing. For a $10 \mathrm{~cm}^{2}$ electrode and $1 \mathrm{~mm}$ distance, assuming a relative dielectric constant of 1.4 (that of cotton [11]) yields $C_{\mathrm{e}} \approx$ $12 \mathrm{pF}$, and if $R_{\mathrm{B}}=100 \mathrm{G} \Omega, f_{\mathrm{c}} \approx 0.1 \mathrm{~Hz}$. Therefore, large percent changes in $C_{\mathrm{e}}$ could be acceptable. However, $C_{\mathrm{e}}$ and $C_{\mathrm{i}}$ will form a voltage divider and the voltage loading effect that results because the condition $C_{\mathrm{e}} \gg C_{\mathrm{i}}$ is not fulfilled, can yield a variable signal attenuation.

Moreover, if the subject is ungrounded, electrostatic 
charges can also yield a signal synchronous with the respiration and the heart beat [4].

In summary, microphonics can modulate the amplitude of body surface potentials recorded by capacitive electrodes regardless of whether those potentials have a bioelectric or a triboelectric origin, and the modulating signal will be that describing the distance variation between electrode plates, that can have a respiratory or cardiac origin, in addition to voluntary body movements.

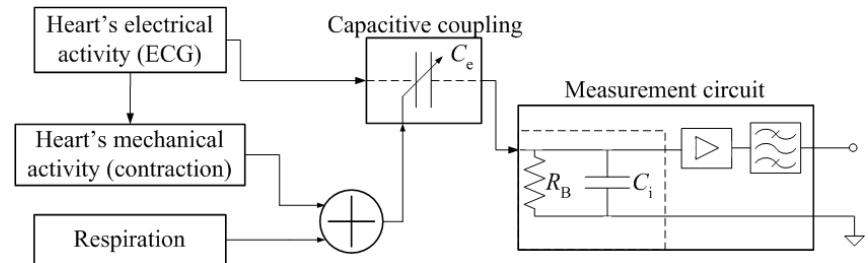

Fig. 1. Capacitive ECG recordings from the backrest of a seat are affected by respiration and mechanical activity of the heart because these make the electrode capacitance $C_{\mathrm{e}}$ to change.

\section{MATERIALS AND METHODS}

\section{A. Capacitive active electrodes}

We built two rectangular electrodes $(10 \mathrm{~cm} \times 5 \mathrm{~cm})$ from conductive tape and attached them to the front side of the backrest of an office chair. Both electrodes were on the same horizontal line, with their centers at $16 \mathrm{~cm}$ from each other, and symmetrically placed with respect to an imaginary vertical line running along the backrest, and presumably close to the vertebral column of a seated subject. The height of the electrodes with respect to the seat roughly corresponded to that of the seventh thoracic vertebra.

Each electrode was connected to a voltage buffer built from an OPA129 op amp, whose input impedance is $10^{13} \Omega$ $\left(R_{\mathrm{i}}\right)$ shunted by $1 \mathrm{pF}\left(C_{\mathrm{i}}\right)$. The typical input bias current is $\pm 30 \mathrm{fA}$ and to provide a bias path, a resistor $R_{\mathrm{B}}=11 \mathrm{G} \Omega$ was connected between the non-inverting op amp input and signal common (signal ground). Hence, for a $1 \mathrm{~mm}$ distance between capacitor "plates", $C_{\mathrm{e}} \approx 60 \mathrm{pF}$ and $f_{\mathrm{c}} \approx 0.2 \mathrm{~Hz}$.

\section{B. Assessment of distance changes in capacitive electrodes}

To evaluate the variation in electrode capacitance because of distance changes between the body and each electrode, we devised the system in Fig. 2. A safe ac voltage $\left(v_{\mathrm{S}}\right)$ is applied to the body and the output $v_{\mathrm{O}}$ of the voltage buffer is recorded. Any amplitude modulation experienced by the injected voltage will reflect the varying attenuation of the voltage divider formed by $C_{\mathrm{e}}$ and $C_{\mathrm{i}}$. Demodulating $v_{\mathrm{O}}$ provides insight into the origin of those distance variations.

We applied a $3 \mathrm{~V}$ peak-to-peak $50 \mathrm{kHz}$ sinusoidal voltage to the left arm of the subject and measured the signal at the output of the voltage buffer connected to the electrode closer to the heart. The signal was high-pass filtered before demodulating it, amplified $(G=1000)$, and band-pass filtered $(0.16 \mathrm{~Hz}-5 \mathrm{~Hz})$, as shown in Fig. 3.
For reference, cardiac and respiratory activity was simultaneously monitored by a piezoelectric film attached to the back side of the chair's backrest. Forces resulting from thoracic movements induced by respiration and cardiac activity are transmitted to surfaces in contact with the body. These forces stress the backrest and the piezoelectric sensor yields an output voltage proportional to the force signal.

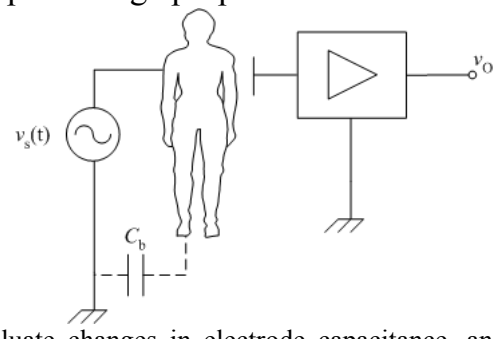

Fig. 2. To evaluate changes in electrode capacitance, an ac voltage was applied to the body and the buffer output was synchronously demodulated.

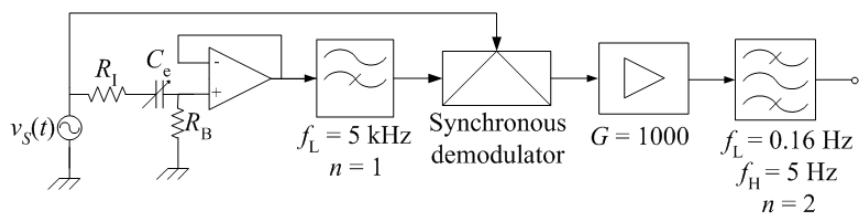

Fig. 3. Block diagram of the circuits to detect and demodulate the signal from capacitive electrodes whose capacitance is modulated by cardiac and respiratory activity. $R_{\mathrm{I}}$ is the internal body resistance.

\section{Respiratory- and cardiac-related movements in ECG recordings with capacitive electrodes}

Fig. 4 shows the differential amplifier we built to acquire the ECG with capacitive electrodes. A differential first-order $\mathrm{HPF}$ at $0.16 \mathrm{~Hz}$ cancels offset voltages from the voltage buffers and attenuates their drifts. The ECG signal is amplified $(G=2005)$ by an instrumentation amplifier (IA), whose output is fed back to its reference terminal to build and additional HPF. A Butterworth third-order low-pass filter limits the measurement bandwidth to $100 \mathrm{~Hz}$.

To reduce power line interference, the backrest and the voltage buffers were covered with conductive material connected to signal common to act as a shield. As a reference, the ECG was also obtained with two pre-gelled conductive electrodes; no reference ("right leg") electrode was used, which excluded any influence from this amplifier on the ECG signal obtained by the capacitive electrodes. The system was supplied by batteries and the ECG amplifiers (for capacitive and conductive electrodes) had separate voltage regulators. Distance changes in capacitive electrodes and the biopotential signals were measured for a rigid and for a foam-covered backrest. All signals were digitized at 1000 samples per second and 12 bit resolution. Changes in electrode capacitance were digitized by an isolated-input DL750 scope recorder (Yokogawa), which was also used in the first set of capacitive "ECG" recordings. ECG signals from the foam-covered backrest, were digitized by a data acquisition module (Eagle) controlled by a portable PC. This offered better isolation than the line-powered recorder. Measurements were carried 
out in two people at different times. All measurements shown here belong to the same person.

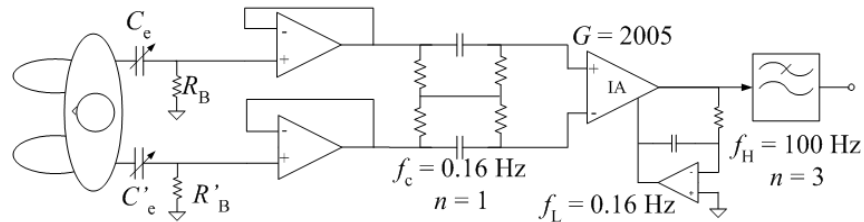

Fig. 4. ECG amplifier for capacitive electrodes placed on the backrest of an office chair.

\section{EXPERIMENTAL RESULTS AND DISCUSSION}

\section{A. Capacitance variations in electrodes}

The lower trace in Fig. 5 shows that the force signal in the backrest mostly results from the respiration, and that it includes components that can be attributed to cardiac activity. The upper trace shows cyclic variations of the demodulated amplitude of the output of the voltage buffer connected to the left-side electrode; hence, they reflect distance variations. These variations coincide with those of the force due to the respiration and, superimposed on them, there are some spikes synchronous with the cardiac activity as revealed by similar spikes in the force signal. The spectra of both signals, shown in Fig. 6, corroborate that the force exerted on the backrest and the variations in electrode capacitance $\left(C_{\mathrm{e}}\right)$ are probably related. This variation in electrode capacitance could explain the abnormal ECG waveforms recorded in [8], and the extra peaks in the ECG in [12], both recorded with capacitive electrodes.

The output of the voltage buffer connected to the rightside electrode displayed variations similar to those in the left-side electrode.

\section{B. Respiration and cardiac-related movements in capacitive ECG recordings}

Fig. 7 shows, from top down, the conventional ECG (conductive electrodes), surface biopotential measured with capacitive electrodes (capacitive ECG) and the force signal on the rigid chair's backrest. The wandering of the center signal follows the force signal (bottom trace) and its small spikes seem to coincide with QRS complexes in the ECG. Some additional spikes in the center signal seem to be related to those spikes in the force signal that, because they appear after the QRS on a quite regular basis, could be related to the BCG. The spectra of the force signal and the capacitively-coupled biopotential signal (Fig. 8) are mostly attributable to respiration. Recordings obtained in other sessions displayed a preponderance of BCG-induced spikes over those coincident with the QRS complex.

From Fig. 8, microphonics during normal breathing conditions will mostly have a low frequency contents $(<0.5 \mathrm{~Hz})$. Therefore, a HPF at $0.5 \mathrm{~Hz}$ will reduce that effect on the ECG obtained with capacitive electrodes. This was in fact the case in [6] and [13], where the respective corner frequencies of the HPF formed by the electrode and the bias resistor of the voltage buffer were about $1.8 \mathrm{~Hz}$ and
$3 \mathrm{~Hz}$. In addition, in [13] a fourth-order HPF with $f_{\mathrm{c}}=$ $0.5 \mathrm{~Hz}$ was used. These filters, however, also attenuate the ECG signal. If the signal does not saturate any stage because of excessive interference, digital filters can yield a clean ECG signal [8]. Furthermore, in long-term measurements, perspiration can reduce microphonics [9], as corroborated in [8], but unavoidable relative movements between the body and electrodes result in large artifacts.
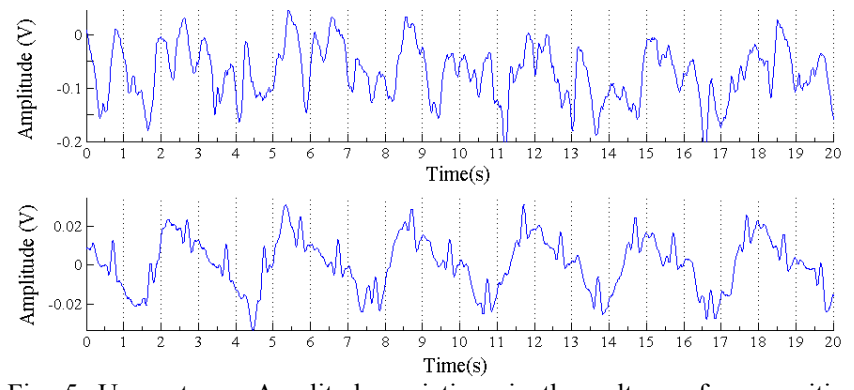

Fig. 5. Upper trace: Amplitude variations in the voltage of a capacitive electrode as obtained by the method in Fig. 2 and the circuit in Fig. 3. Lower trace: Force signal measured in the chair's backrest.

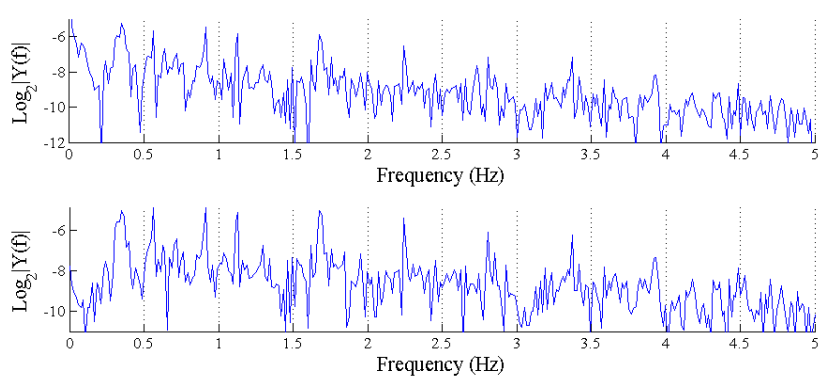

Fig. 6. Spectra of the capacitively-coupled signal (upper trace) and force signal (lower trace). The main components in both spectra are due to respiration.

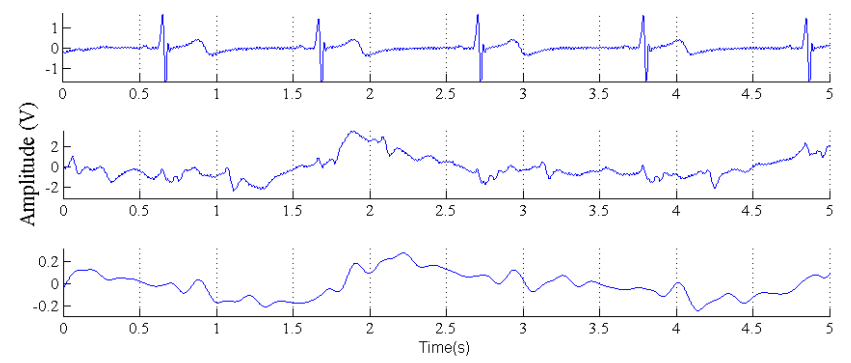

Fig. 7. From the top: conventional ECG with two electrodes, capacitive "ECG", and force signal. The center signal looks more like the force signal than like the ECG.

If capacitive electrodes are fixed on a foam-covered backrest, relative movements between the body surface and the electrodes should be smaller as the electrodes better adapt to the torso surface. The upper trace in Fig. 9 shows the electrode displacement signal, whose measurement required additional amplifier $\left(G_{2}=4\right)$ and band-pass filter $(0.16 \mathrm{~Hz}$ to $5 \mathrm{~Hz})$ stages. Compared to Fig. 5, there is a negligible respiratory contribution and only some peaks appear to coincide with the cardiac activity in the force signal (lower trace). Fig. 10 shows that the capacitive ECG (middle trace) is not affected by respiration either. The main difference with respect to the conventional ECG is the 
amplitude, because the gain for this last one was 1340 . Capacitive ECG is also more susceptible to power-line interference, probably because of the higher electrode impedance.
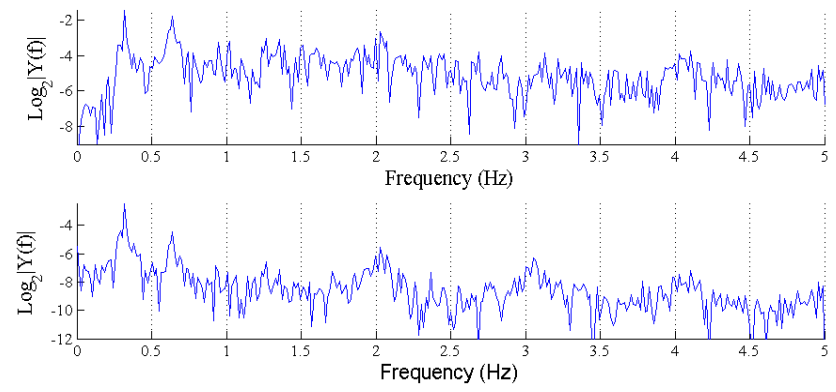

Fig. 8. The main spectral contents of capacitively-coupled potential measurements (upper trace) and force signal (lower trace) are attributable to respiration. Cardiac-related activity can be barely distinguished.

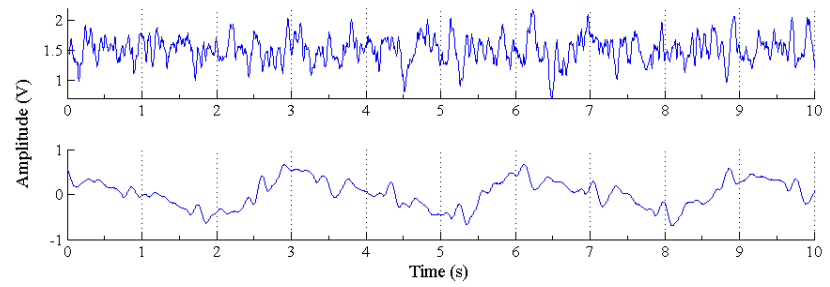

Fig. 9. Distance variations in a capacitive electrode placed on a soft backrest (upper trace) are less susceptible to respiration and cardiac activity than those in a rigid backrest. Lower trace: Simultaneously measured force signal.

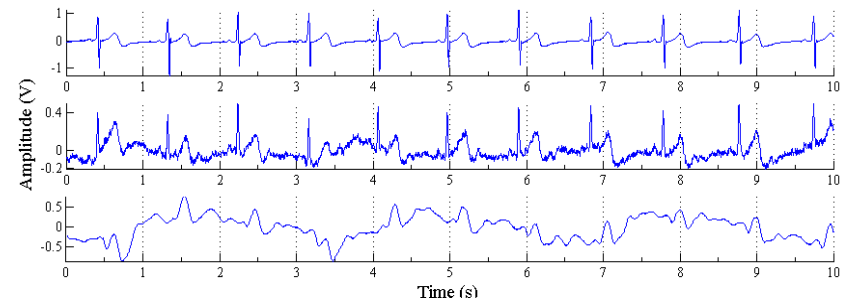

Fig. 10. From the top: conventional ECG with two conductive electrodes, capacitive ECG and force signal in the chair's backrest. Microphonics decreases when capacitive electrodes are on a soft chair's backrest.

\section{CONCLUSION}

Microphonics is any electrical interference caused by mechanical vibration. When measuring bioelectric signals, those vibrations can be due to physiological processes such as breathing or cardiac activity. When measuring biopotentials using capacitive electrodes, body vibrations make the value of the capacitor formed by the skin and the metal electrode to change. Thus, microphonics will modify the biopotential waveform to the extent that the bioelectric signal can be completely masked, for example by respiratory movements.

We have shown that the capacitance of electrodes placed on the rigid backrest of a seat changes mostly because of the respiration and the BCG (Fig. 5). Microphonics can thus mask QRS complexes in ECGs obtained with capacitive electrodes that are not attached to the body. High-pass filtering could reduce the effect of respiratory movements on ECGs obtained with capacitive electrodes, but this would also affect the ECG signal. Also, depending on the position of the electrodes relative to the heart, distance variations due to the BCG can distort the ECG signal because they result in spikes after QRS complexes. Those ECGs could be difficult to analyze for diagnostic purposes.

A soft backrest better adapts to the body surface and distance variation between electrodes and body because of respiration are greatly reduced (Fig. 9). Distance variations because of the beating heart are also reduced. As a result, the ECG is less susceptible to microphonics (Fig. 10).

Finally, throughout our experiments we have avoided any connection between systems intended to record the ECG obtained with capacitive electrodes and reference systems, used to obtain the ECG with conventional electrodes, either gelled or dry, as well as any other connection to earth ground. Signals obtained under conditions other than these may display a behavior different to that here reported.

\section{REFERENCE}

[1] C. J. Harland, T. D. Clark, R. J. Prance, "Electric potential probes-new directions in the remote sensing of the human body," Meas. Sci. Technol. vol. 13, pp. 163-169, 2002.

[2] W. J. Smith and J. R. LaCourse, "Non-contact biopotential measurement from the human body using a low-impedance charge amplifier," in Bioengineering Conference. IEEE 30th Annual Northeast, 2004, pp. 31-32.

[3] P. C. Richardson and R. M. Adams, "Electric-field disturbances near the human body," J. Appl. Physiol., vol. 26, pp. 838-840, 1969.

[4] O. Casas and R. Pallas-Areny, "Electrostatic interference in contactless biopotential measurements," in IEEE-EMBS Conference, 2007, pp. 2655-2658.

[5] C. J. Harland, T. D. Clark and R. J. Prance, "High resolution ambulatory electrocardiographic monitoring using wrist-mounted electric potential sensors," Meas. Sci. Technol. vol. 14, 923-928, 2003.

[6] Yong Gyu Lim, Ko Keun Kim and S. Park, "ECG measurement on a chair without conductive contact," IEEE Trans Biomed. Eng., vol. 53, pp. 956-9, 05. 2006.

[7] M. Steffen, A. Aleksandrowicz and S. Leonhardt, "Mobile Noncontact Monitoring of Heart and Lung Activity," IEEE Trans Biomedical Circuits and Systems, vol. 1, pp. 250-257, 2007.

[8] A. Ueno, Y. Akabane, T. Kato, H. Hoshino, S. Kataoka and Y. Ishiyama, "Capacitive Sensing of Electrocardiographic Potential Through Cloth From the Dorsal Surface of the Body in a Supine Position: A Preliminary Study," IEEE Trans Biomed. Eng., vol. 54, pp. 759-766, 2007.

[9] A. Searle and L. Kirkup, "A direct comparison of wet, dry and insulating bioelectric recording electrodes," Physiol. Meas., vol. 21, pp. 271-284, 2000.

[10] A. M. Weissler, Noninvasive Cardiology. Grune \& Stratton, 1974, pp. 1-148.

[11] Clipper Controls Inc. (2005). Dielectric constants guide. [Online] Available: http://clippercontrols.com/info/dielectric_constants.html\#A

[12] Ko Keun Kim, Yong Kyu Lim and Kwang Suk Park, "The electrically noncontacting ECG measurement on the toilet seat using the capacitively-coupled insulated electrodes," IEEE-EMBS Conference, 2004, pp. 2375-2378.

[13] Ko Keun Kim, Yong Kyu Lim and Kwang Suk Park, "Common mode noise cancellation for electrically non-contact ECG measurement system on a chair," in IEEE-EMBS Conference, 2005, pp. 5881-5883. 